Pacific Journal of Mathematic 


\section{ON THE STRUCTURE OF FINITE RINGS II}

\section{ROBERT S. WILSON}

In this paper we develop a structure theory for modules and bimodules over complete matrix rings over Galois rings, and we use this module theory to study the additive structure of the components of a Peirce decomposition of a general finite ring.

We recall that any finite ring is the direct sum of rings of prime power characteristic. This follows from noticing that when one decomposes the additive group of a finite ring into its primary components, the components are ideals of prime power characteristic (cf. [4]). We thus restrict ourselves to considering rings of prime power characteristic without loss of generality up to direct sum formation.

We next recall the definition of a Galois ring. Let $k, r$ be positive integers and $p$ be a prime integer. The Galois ring of characteristic $p^{k}$ and order $p^{k r}$ is defined to be $Z[x] /\left(p^{k}, f(x)\right)$ [8], [10] where $Z$ denotes the rational integers and $f(x) \in Z[x]$ is monic of degree $r$ and irreducible. A Galois ring is uniquely determined up to isomorphism by the integers $p, k$, and $r$, and we shall denote the Galois ring of characteristic $p^{k}$ and order $p^{k r}$ by $G(k, r)$. The prime $p$ will generally be clear from context. Note that $G(1, r) \cong G F\left(p^{r}\right)$ and $G(k, 1) \cong Z /\left(p^{k}\right)$.

If $R$ is a finite ring of characteristic $p^{k}$ which contains a 1 then $R$ contains a Galois ring $G(k, r)$ for some $r$ which contains the 1 of $R$. Indeed $Z /\left(p^{k}\right) \cdot 1$ will always be such a ring. Therefore, any finite ring of characteristic $p^{k}$ is thus a faithful left and right $G(k, r)$-module for some $r$.

We now seek to develop a module theory for matrix rings over Galois rings. In a sense, the theory is already developed in that a matrix ring over a Galois ring is Morita equivalent to a Galois ring and hence the categories of modules will be category isomorphic, and a module and bimodule theory already is known for modules over Galois rings [11]. However, we seek slightly more information than is given by the category isomorphism from Morita theory. In what follows $Q$ will denote the matrix ring $M_{n}(G(k, r))$.

Proposition 1. Let $M$ be a finitely generated left $Q$-module. Then $M$ is a direct sum of cyclic left Q-modules.

Proof. Every finitely generated left $G(k, r)$-module is a direct sum of cyclic left $G(k, r)$-modules by Corollary 2 to Proposition 1.1 of [11]. 
Therefore, by Proposition 2.7 of [9] every finitely generated left $Q$-module is a direct sum of cyclic $Q$-modules.

We have thus reduced the study of $Q$-modules to the study of cyclic $Q$-modules. Let $Q x$ be a cyclic left $Q$-module. Consider the map $q \mapsto q x$ from $Q$ to $Q x$. This map is clearly a $Q$-module homomorphism and thus has a kernel $L$ which is a left ideal of $Q$. We are thus led to consider the left ideals of quasi-simple rings.

Proposition 2. For $j=1, \cdots, n$, let $e_{j}$ denote the matrix whose only nonzero entry is a 1 in the jjth position. Let $L$ be a left ideal in $Q$. Then $L$ is isomorphic to $\sum_{j=1}^{t} p^{i_{j}} Q e_{j}$ for some choice of integers $0 \leqq i_{1}, \cdots, i_{t}<k$ and some $t \leqq n$.

Proof. The proposition boils down to showing that $L$ is isomorphic to a sum of $p^{i}$ th multiples of columns of $M_{n}(G(k, r))$. Let $M$ denote the set of all top rows of matrices in $L . M$ is then, in a natural way a left $G(k, r)$-module and is thus isomorphic to a direct sum of cyclic left $G(k, r)$-modules. Say $M \cong \sum_{j=1}^{t} G(k, r) x_{j}$ where the $x_{j}$ 's are $n$-tuples over $G(k, r)$. In fact they are the top rows of certain matrices in $L$. Note that, since $M$ is contained in a $G(k, r)$-module which is free on $n$ generators, we must conclude that $t \leqq n$. Let $a_{j}$ be the smallest positive integer such that $p^{a_{j}} x_{j}=0$. Note that $0<a_{j} \leqq k$ for all $j=1, \cdots, t$. Now any left ideal of $M_{n}(G(k, r))$ is completely determined by its set of top rows, because to multiply on the left by elements of $M_{n}(G(k, r))$ is to perform operations on the rows of matrices in $L$. Thus it follows that $L \cong \sum_{j=1}^{t} p^{k-a_{j}} Q e_{j}$, since the set of top rows of the ideal on the right is isomorphic to the set of top rows of $L$.

Proposition 3. Any finitely generated left Q-module is isomorphic to a direct sum of $p^{j}$ th multiples of columns of $M_{n}(G(k, r))$. Moreover, any finitely generated indecomposable left $Q$-module is isomorphic to a $p^{j}$ th multiple of a column of $M_{n}(G(k, r))$.

Proof. From Proposition 1 it suffices to prove the result for cyclic left modules. As noted above a cyclic module is isomorphic to $Q / L$ for some left ideal $L$. Apply Proposition 2 and let $L \cong \sum_{i=1}^{n} p^{j_{i}} Q e_{i}$ where $e_{i}$ is the element of $Q$ corresponding to the matrix in $M_{n}(G(k, r))$ which has a 1 in the $i$ ith position and 0 's elsewhere. Now $0 \leqq j_{1}$, $\cdots, j_{n} \leqq k$ so define $M=\sum_{i=1}^{n} p^{k-j_{i}} Q e_{i}$. It is easy to see that $M \cong Q / L$, and $Q e_{i}$ is isomorphic to a column in $M_{n}(G(k, r))$.

To see that any finitely generated indecomposable left module is isomorphic to a $p^{j}$ th multiple of a column of $M_{n}(G(k, r))$, let $M$ be a finitely generated indecomposable left $Q$-module. Then being finitely 
generated it is the sum of a finite number of modules isomorphic to $p^{j}$ th multiples of columns of $M_{n}(G(k, r))$. But clearly any column of $M_{n}(G(k, r))$ is indecomposable. Therefore, applying the Krull-Schmidt theorem we conclude that the decomposition of $M$ as a sum of $p^{j}$ th multiples of columns of $M_{n}(G(k, r))$ consists of one $p^{j}$ th column of $M_{n}(G(k, r))$ and we are done.

We next turn our attention to bimodules over matrix rings over Galois rings. Let $Q_{1}, Q_{2}$ be two such rings. If $M$ is a $\left(Q_{1}, Q_{2}\right)$-module then it is a left $Q_{1} \otimes_{z} Q_{2}^{o p}$-module where $Q_{2}^{o p}$ is a ring which has the same additive group as $Q_{2}$ but in which multiplication is defined by $a \cdot b=b a$, the product on the right being taken in $Q_{2}$. But $Q_{2}$ is a matrix ring over a commutative ring and matrix rings over commutative rings are anti-isomorphic to themselves via the transpose map.

We now consider the tensor product of matrix rings over Galois rings.

Proposition 4. Let $Q_{1}=M_{n_{1}}\left(G\left(k_{1}, r_{1}\right)\right), Q_{2}=M_{n_{2}}\left(G\left(k_{2}, r_{2}\right)\right)$. Let $d=\operatorname{gcd}\left\{r_{1}, r_{2}\right\}, k=\min \left\{k_{1}, k_{2}\right\}, m=\operatorname{lcm}\left\{r_{1}, r_{2}\right\}$.

Then

$$
Q_{1} \bigotimes_{Z} Q_{2} \cong \sum_{1}^{d} \cdot M_{n_{1} n_{2}}(G(k, m))
$$

Proof. In order to prune the hanging gardens of subscripts in what follows we shall denote $Z /\left(p^{k}\right)$ by $K$. We first note that

$$
M_{n_{1}}\left(G\left(k_{1}, r_{1}\right)\right) \bigotimes_{Z} M_{n_{2}}\left(G\left(k_{2}, r_{2}\right)\right) \cong M_{n_{1}}\left(G\left(k, r_{1}\right)\right) \bigotimes_{K} M_{n_{2}}\left(G\left(k, r_{2}\right)\right) .
$$

Thus

$$
\begin{aligned}
Q_{1} \bigotimes_{Z} Q_{2} & \cong M_{n_{1}}\left(G\left(k, r_{1}\right)\right) \bigotimes_{K} M_{n_{2}}\left(G\left(k, r_{2}\right)\right) \\
& \cong M_{n_{1}}(K) \bigotimes_{K} G\left(k, r_{1}\right) \bigotimes_{K} G\left(k, r_{2}\right) \bigotimes_{K} M_{n_{2}}(K) \\
& \cong M_{n_{1}}(K) \bigotimes_{K} \sum_{1}^{d} \cdot G(k, m) \bigotimes_{K} M_{n_{2}}(K)
\end{aligned}
$$

(by Proposition 1.2 of [11])

$$
\begin{aligned}
& \cong \sum_{1}^{d} \cdot\left(G(k, m) \bigotimes_{K} M_{n_{1}}(K) \bigotimes_{K} M_{n_{2}}(K)\right) \\
& \cong \sum_{1}^{d} \cdot\left(G(k, m) \bigotimes_{K} M_{n_{1} n_{2}}(K)\right) \\
& \cong \sum_{1}^{d} \cdot M_{n_{1} n_{2}}(G(k, m)) .
\end{aligned}
$$

We are now able to obtain a description of $\left(Q_{1}, Q_{2}\right)$-modules where $Q_{1}$ and $Q_{2}$ are matrix rings over Galois rings. 
PROPOSITION 5. Let $Q_{1}=M_{n_{1}}\left(G\left(k_{1}, r_{1}\right)\right), Q_{2}=M_{n_{2}}\left(G\left(k_{2}, r_{2}\right)\right)$ and $k=\min \left\{k_{1}, k_{2}\right\}, m=\operatorname{lcm}\left\{r_{1}, r_{2}\right\} . M$ is a $\left(Q_{1}, Q_{2}\right)$-module. Then $M$ is of the form

$$
M \cong \sum_{i=1}^{n} p^{j_{i}} M_{n_{1}, n_{2}}(G(k, m))
$$

where $M_{n_{1}, n_{2}}(G(k, m))$ denotes the set of $n_{1} \times n_{2}$ matrices over $(G(k, m))$.

Proof. It is instructive to first ask how $M_{n_{1}}\left(G\left(k_{1}, r_{1}\right)\right)$ acts as a ring of left operators on $M_{n_{1}, n_{2}}(G(k, m))$ and how $M_{n_{2}}\left(G\left(k_{2}, r_{2}\right)\right)$ acts as a ring of right operators on $M_{n_{1}, n_{2}}(G(k, m))$. Well, since

$$
G\left(k_{1}, r_{1}\right) \bigotimes_{z} G\left(k_{2}, r_{2}\right) \cong \sum_{1}^{d} \cdot G(k, m)
$$

where $d=\operatorname{gcd}\left\{r_{1}, r_{2}\right\}$ by Proposition 1.2 of [11] it follows that $G\left(k_{1}, r_{1}\right)$ acts as a ring of left operators and that $G\left(k_{2}, r_{2}\right)$ acts as a ring of right operators on $G(k, m)$. We can thus impose a $\left(Q_{1}, Q_{2}\right)$-module structure on $M_{n_{1}, n_{2}}(G(k, m))$ by defining

$$
\left[a_{i j}\right]\left[b_{i j}\right]=\left[\sum_{q=1}^{n_{1}} a_{i q} b_{q j}\right]
$$

if $\left[a_{\imath j}\right] \in M_{n_{1}}\left(G\left(k_{1}, r_{1}\right)\right),\left[b_{i j}\right] \in M_{n_{1}, n_{2}}(G(k, m))$ and

$$
\left[b_{i j}\right]\left[c_{i j}\right]=\left[\sum_{q=1}^{n_{2}} b_{i q} c_{q j}\right]
$$

if $\left[b_{i j}\right] \in M_{n_{1}, n_{2}}(G(k, m))$ and $\left[c_{i j}\right] \in M_{n_{2}}\left(G\left(k_{2}, r_{2}\right)\right)$.

Now let $M$ be a $\left(Q_{1}, Q_{2}\right)$-module. Then $M$ can be considered as a $Q_{1} \otimes_{Z} Q_{2}^{o p}$-module and as $Q_{2}^{o p} \cong Q_{2}$ it can be considered as a left $Q_{1} \otimes_{Z} Q_{2}$-module. Let $e_{1}, \cdots, e_{d}$ be a complete set of orthogonal primitive central idempotents for $\sum_{1}^{\cdot d} M_{n_{1} n_{2}}(G(k, m))$. Then since $e_{1}+\cdots+e_{d}=1, M=1 M=\left(e_{1}+\cdots+e_{d}\right) M=e_{1} M+\cdots+e_{d} M$ and this sum is direct since the $e_{i}$ are orthogonal idempotents. Moreover, each $e_{i} M$ is a left $M_{n_{1} n_{2}}(G(k, m))$-module. We then conclude that $M$ is isomorphic to a direct sum of $p^{j}$ th multiples of columns of the component matrices $\sum_{1}^{\cdot d} M_{n_{1} n_{2}}(G(k, m))$.

It thus suffices to show that a column of a component matrix in $\sum_{1}^{\cdot d} M_{n_{1} n_{2}}(G(k, m))=Q_{1} \bigotimes_{Z} Q_{2}^{o p}$ is isomorphic to $M_{n_{1}, n_{2}}(G(k, m))$ as a $\left(Q_{1}, Q_{2}\right)$-module. We first note the isomorphism from $M_{n_{1}}\left(G\left(k_{1}, r_{1}\right)\right) \otimes_{z}$ $M_{n_{2}}\left(G\left(k_{2}, r_{2}\right)\right)$ into

$$
\sum_{1}^{d} \cdot M_{n_{1} n_{2}}(G(k, m))=M_{n_{1} n_{2}}\left(\sum_{1}^{d} \cdot G(k, m)\right)=M_{n_{1} n_{2}}\left(G\left(k_{1}, r_{1}\right) \bigotimes_{Z}\left(G\left(k_{2}, r_{2}\right)\right)\right.
$$

is defined by $\left[a_{i j}\right] \otimes\left[b_{p q}\right] \rightarrow$ 


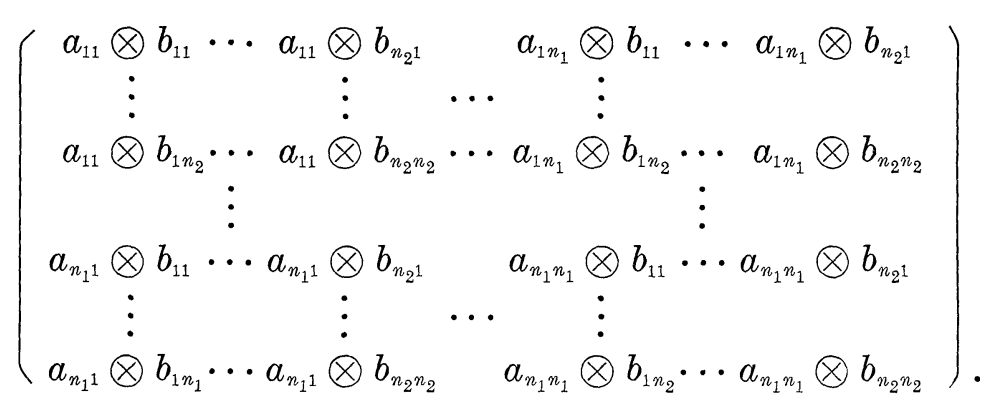

One can check by straightforward computation that a column in this matrix ring, i.e., something of the form

$$
\left(\begin{array}{c}
a_{1 j} \otimes b_{p 1} \\
\vdots \\
a_{1 j} \otimes b_{p n_{2}} \\
\vdots \\
a_{n_{1 j}} \otimes b_{p 1} \\
\vdots \\
a_{n_{1 j}} \otimes b_{p n_{2}}
\end{array}\right)
$$

is isomorphic to the matrix whose $i q$ th entry is $a_{i j} \otimes b_{p q}$ as a $\left(Q_{1}, Q_{2}\right)$ module. But

$$
A=\left\{\left(\begin{array}{c}
a_{1 j} \otimes b_{p 1} \\
\vdots \\
a_{1 j} \otimes b_{p n_{2}} \\
\vdots \\
a_{n_{1 j}} \otimes b_{p 1} \\
\vdots \\
a_{n_{1 j}} \otimes b_{p n_{2}}
\end{array} \mid a_{\cdot j} \in G\left(k_{1}, r_{1}\right), b_{p q} \in G\left(k_{2}, r_{2}\right)\right\}\right.
$$

has a decomposition as a sum of indecomposable modules as a direct sum of $d$ columns of $M_{n_{1}, n_{2}}(G(k, m))$ and the $\left(Q_{1}, Q_{2}\right)$-module $M_{n_{1}, n_{2}}\left(G\left(k_{1}, r_{1}\right)\right) \bigotimes_{Z}\left(G\left(k_{2}, r_{2}\right)\right)$ which is isomorphic to $A$ has a decomposition as a sum of indecomposable $\left(Q_{1}, Q_{2}\right)$-modules as a direct sum of $d$ copies of $M_{n_{1}, n_{2}}(G(k, r))$. The Krull-Schmidt theorem tells us then that a column of a component matrix in $\sum_{1}^{\cdot d} M_{n_{1} n_{2}}(G(k, m))$ is isomorphic as a $\left(Q_{1}, Q_{2}\right)$-module to $M_{n_{1}, n_{2}}(G(k, m))$.

We now apply these results to the study of the additive structure of an arbitrary finite ring. But in order to do this we must first obtain the existence of a subring of our ring, which is a direct sum of matrix rings over Galois rings and which contains all of the idempotents. The existence of such a subring and its uniqueness up 
to inner automorphism follows directly from Theorem 33 of [1] which was viewed by Azumaya as a generalization of the WedderburnMalcev theorem [3; $\$ 72.19]$. In addition Clark [2] recently proved the existence of such a subring of a finite ring using elementary methods. However, in the case of a finite ring more can be said about this subring than existence and uniqueness up to inner automorphism. Specifically we have:

Proposition 6. Let $R$ be a finite ring with 1 of characteristic $p^{k}$ and radical $J$. Then $R$ contains a subring $Q$ isomorphic to a direct sum of matrix rings over Galois rings such that $Q / p Q \cong R / J$ and $a(Q, Q)$-submodule $M$ of $J$ such that $R=Q+M$ with $Q \cap M=\{0\}$.

REMARK. Once we have the existence of $Q$ it is immediate that $Q$ is a direct summand of $R$ when $R$ is considered either as a left or a right $Q$-module because $Q$ is quasi-Frobenius. However, it does not seem immediately obvious that a complementary left $Q$ direct summand will be a right $Q$-module or that any complementary module can be chosen to be contained in $J$.

Proof of Proposition 6. Suppose $R / J \cong \sum_{i=1}^{m} M_{n_{i}}\left(G F\left(p^{r_{i}}\right)\right)$ and let $\overline{e_{i}}$ be the multiplicative identity of the simple component of $R / J$ isomorphic to $M_{n_{i}}\left(G F\left(p^{r_{i}}\right)\right)$. Then $\overline{e_{1}}, \cdots, \overline{e_{m}}$ is a finite set of orthogonal idempotents in $R / J$. Let $e_{1}, \cdots, e_{m}$ be orthogonal idempotents of $R$ such that $e_{i}+J=\overline{e_{i}}$, and such that $e_{1}+\cdots+e_{m}=1$ (Proposition 5 on p. 54 of [7]). Consider the Peirce decomposition of $R$ with respect to this set of orthogonal idempotents.

$$
R=\sum_{i=1}^{m} e_{i} R e_{i}+\sum_{i \neq j} e_{i} R e_{j} .
$$

As is easy to check each $e_{i} R e_{j}$ is a left $\sum_{i=1}^{m} e_{i} R e_{i}$-module and a right $\sum_{i=1}^{m} e_{i} R e_{i}$-module so this is a $\left(\sum_{i=1}^{m} e_{i} R e_{i}, \sum_{i=1}^{m} e_{i} R e_{i}\right)$-module direct sum decomposition of $R$. Now, as in the proof of Theorem 2 on p. 56 of [7] the $e_{i} R e_{i}$ are primary rings which annihilate each other in pairs and for all $i \neq j e_{i} R e_{j} \subset J$. Since each $e_{i} R e_{i}$ is primary, again using Theorem 1 of p. 56 of [7] we have that each $e_{i} R e_{i}$ is isomorphic to a complete matrix ring over a completely primary ring $C_{i}, e_{i} R e_{i} / e_{i} J e_{i} \cong$ $M_{n_{i}}\left(G F\left(p^{r_{i}}\right)\right)$ so by lifting idempotents again we conclude that $e_{i} R e_{i} \cong$ $M_{n_{i}}\left(C_{i}\right)$. Let $J_{i}$ be the radical of $C_{i}$ with $C_{i} / J_{i} \cong G F\left(p^{r_{i}}\right)$, and the characteristic of $C_{i}$ be $p^{k_{i}}$. Then by Theorem 8 of [10] we have that $C_{i}$ contains a subring isomorphic to $G\left(k_{i}, r_{i}\right)$, we define $Q=\sum \dot{m}_{i=1}^{m} M_{n_{i}}\left(G\left(k_{i}, r_{i}\right)\right)$. Now by Proposition 2.2 of [11] each $C_{i}$ contains a $\left(G\left(k_{i}, r_{i}\right)\right),\left(G\left(k_{i}, r_{i}\right)\right)$-submodule $N_{i}$ with $N_{i} \subset J_{i}$ such that $C_{i}=$ $G\left(k_{i}, r_{i}\right)+N_{i}$. Thus $e_{i} R e_{i}=M_{n_{i}}\left(C_{i}\right)=M_{n_{i}}\left(G\left(k_{i}, r_{i}\right)\right)+M_{n_{i}}\left(N_{i}\right)$ with 
$M_{n_{i}}\left(N_{i}\right)$ a $\left(M_{n_{i}}\left(G\left(k_{i}, r_{i}\right)\right)\right), M_{n_{i}}\left(G\left(k_{i}, r_{i}\right)\right)$-submodule of $M_{n_{i}}\left(C_{i}\right)$. Since the $e_{i} R e_{i}$ annihilate each other in pairs we conclude that each $M_{n_{i}}\left(N_{\imath}\right)$ is a $(Q, Q)$-submodule. Now each $e_{\imath} R e_{j}$ is a $\left(\sum_{i=1}^{m} e_{i} R e_{i}, \sum_{i=1}^{m} e_{\imath} R e_{i}\right)$-submodule so it is a fortiori a $(Q, Q)$-submodule, and we have the following $(Q, Q)$-module direct sum decomposition.

$$
R=Q+\sum_{i=1}^{m} \cdot M_{n_{i}}\left(N_{\imath}\right)+\sum_{i \neq i} \cdot e_{i} R e_{j}
$$

with $M=\sum_{i=1}^{m} M_{n_{i}}\left(N_{\imath}\right)+\sum_{i \neq j} e_{i} R e_{j} \subset J$. Moreover, $Q=\sum_{i=1}^{m} M_{n_{i}}\left(G\left(k_{i}, r_{i}\right)\right)$ and $p Q=\sum_{i=1} M_{n_{i}}\left(p G\left(k_{i}, r_{i}\right)\right)$. Hence

$$
\begin{aligned}
Q / p Q & =\sum_{i=1}^{m} M_{n_{i}}\left(G\left(k_{i}, r_{i}\right)\right) / \sum_{i=1}^{m} M_{n_{i}}\left(p G\left(k_{i}, r_{i}\right)\right) \\
& \cong \sum_{i=1}^{m} M_{n_{i}}\left(G\left(k_{i}, r_{i}\right) / p G\left(k_{i}, r_{i}\right)\right) \\
& \cong \sum_{i=1}^{m} M_{n_{i}}\left(G F\left(p^{r_{i}}\right) \cong R / J .\right.
\end{aligned}
$$

In the classical Wedderburn-Malcev theorem we have $R=S+J$ where $S$ is semi-simple and $S \cap J=\{0\}$. The question arises: in the decomposition we obtained, $R=Q+M$ can we take $M=J$ ? Well $M \subset J$ so surely $R=Q+J$. However, one can see that $Q \cap J=p Q$ and so if $Q \cap J=(0)$ then the characteristic of $Q$, hence of $R$ is $p$, since $Q$ contains the multiplicative identity of $R$. So we ask instead, can we assume that $M$ is an ideal of $R$, or at least a subring? First we note that since $R=Q+M$ and $M$ is a $(Q, Q)$-submodule of $R$, that $M$ will be a two-sided ideal of $R$ if and only if it is a subring of $R$. If the characteristic of $R$ is $p$ then $R$ is an algebra over the field $Z /(p)$, and since any finite extension of a finite field is a separable extension, the hypotheses of the classical Wedderburn-Malcev theorem are satisfied and the answer is yes. However, in general the answer is no, as is shown by the following counterexample. Let

$$
R=\left\{\left[\begin{array}{ll}
a & b \\
2 c & d
\end{array}\right] \in M_{2}(Z /(4)) \mid a, b, c, d \in Z /(4)\right\} .
$$

One can check that $R$ is a completely primary finite ring with radical

$$
J=\left\{\left[\begin{array}{ll}
2 a & b \\
2 c & 2 d
\end{array}\right] \in M_{2}(Z /(4)) \mid a, b, c, d \in Z /(4)\right\} .
$$

In this ring we can take

$$
Q=\left\{\left[\begin{array}{ll}
a & 0 \\
0 & a
\end{array}\right] \in M_{2}(Z /(4)) \mid a \in Z /(4)\right\}
$$

and for all invertible $x \in R x^{-1} Q x=Q$. So $M$ is a direct complement 
of $Q, M \subset J$ so every element of $M$ is of the form

$$
\left[\begin{array}{ll}
2 a & b \\
2 c & 2 d
\end{array}\right]
$$

Now

$$
\left[\begin{array}{ll}
0 & 1 \\
2 & 0
\end{array}\right] \in R
$$

so there must be some element of $M$ of the form

$$
\left[\begin{array}{ll}
2 a & 1 \\
2 & 2 a
\end{array}\right]
$$

for some $a \in Z /(4)$. But then

$$
\left[\begin{array}{cc}
2 a & 1 \\
2 & 2 a
\end{array}\right]^{2}=\left[\begin{array}{ll}
2 & 0 \\
0 & 2
\end{array}\right] \in Q
$$

and we conclude that $M$ is not a subring.

Finally, we conclude with remarks on the additive structure of general finite rings of characteristic $p^{k}$. Let $R$ be a finite ring of characteristic $p^{k}$.

Let $J$ be the radical of $R$. Lift the multiplicative identity of $R / J$ to an idempotent $e \in R$. (If $R=J$ then we take $e=0$.) Then $e R e$ is a finite ring of characteristic $p^{j}$ for some $j \leqq k$ and with a multiplicative identity. So we apply Proposition 6 to $e R e$ and obtain a subring $Q$ and a $(Q, Q)$-submodule $N \subset e J e$ which satisfy the properties of Proposition 6. We let $M_{1}=\{e a-e a e \in R \mid a \in R\}, M_{2}=\{a e-$ $e a e \in R \mid a \in R\}$ and $R_{0}=\{a-a e-e a+e a e \in R \mid a \in R\}$. Then $R=$ $e R e \dot{+} M_{1} \dot{+} M_{2} \dot{+} R_{0}$ is an (eRe,eRe)-module direct sum decomposition hence a fortiori a $(Q, Q)$-module decomposition of $R$. We consider $R_{0}$ as a $\left(Z /\left(p^{k}\right), Z /\left(p^{k}\right)\right)$-module, $M_{1}$ as a right $Z /\left(p^{k}\right)$-module and $M_{2}$ as a left $Z /\left(p^{k}\right)$-module via the module structures they inherit as additive subgroups of a ring of characteristic $p^{k}$. We then let $\bar{Q}=Q+Z /\left(p^{k}\right)$ and define a $(\bar{Q}, \bar{Q})$-module structure on $R$ by $(q, z)\left(r_{1}+m_{1}+m_{2}+r_{0}\right)=$ $q r_{1}+q m_{1}+z m_{2}+z r_{0}$ and $\left(r_{1}+m_{1}+m_{2}+r_{0}\right)(q, z)=r_{1} q+m_{1} z+m_{2} q+$ $r_{0} z$ where $q \in Q, z \in Z /\left(p^{k}\right), r_{1} \in e R e, m_{1} \in M_{1}, m_{2} \in M_{2}$, and $r_{0} \in R_{0}$. Then the decomposition $R=e R e+M_{1}+M_{2}+R_{0}$ is a $(\bar{Q}, \bar{Q})$-module direct sum decomposition, $M_{1}$ is a $\left(Q, Z /\left(p^{k}\right)\right)$-module $M_{2}$ is a $\left(Z /\left(p^{k}\right), Q\right)$-module and $R_{0}$ is a nilpotent subring which is also a $\left(Z /\left(p^{k}\right), Z /\left(p^{k}\right)\right)$-submodule. If $e R e=Q+N$ is the decomposition given by Proposition 6 then if we define $\bar{N}=N+M_{1}+M_{2}+R_{0}$ then $R=Q+\bar{N}$ is a $(\bar{Q}, \bar{Q})$-module direct sum decomposition of $R$ into a quasi-semi-simple ring and a $(\bar{Q}, \bar{Q})$-submodule of $J$. 
We thus have a Peirce decomposition of a general finite ring of characteristic $p^{k}$

$$
\begin{aligned}
R= & \sum_{i=1}^{m} e_{i} R e_{\imath}+\sum_{i \neq j}^{m} e_{i} R e_{j}+\sum_{i=1}^{m}(1-e) R e_{\imath} \\
& +\sum_{i=1}^{m} e_{i} R(1-e)+(1-e) R(1-e),
\end{aligned}
$$

where $e_{1}, \cdots, e_{m}$ are a complete set of orthogonal idempotents which are central modulo the radical, and where $e_{1}+\cdots+e_{m}=e$. The $e_{i} R e_{i}$ are matrix rings over completely primary finite rings and completely primary finite rings were studied in $\S 3$ of [11]. If the completely primary finite rings of $e_{i} R e_{i}$ is $C_{\imath}$ and its radical is $J_{\imath}$ with $C_{2} / J_{i} \cong G F\left(p^{r_{i}}\right)$ and the characteristic of $C_{i}$ is $p^{k_{i}}$ and $e_{2} R e_{i} \cong M_{n_{i}}\left(C_{i}\right)$, then $e_{i} R e_{j}$ is a $\left(M_{n_{i}}(G)\left(k_{i}, r_{i}\right)\right), M_{n_{j}}\left(G\left(k_{j}, r_{j}\right)\right)$-module, and the structure of such modules was studied in Propositions 1-5. Each $(1-e) R e_{\imath}$ is a right $M_{n_{i}}\left(G\left(k_{i}, r_{\imath}\right)\right)$-module and each $e_{\imath} R(1-e)$ is a left $M_{n_{i}}\left(G\left(k_{i}, r_{\imath}\right)\right)$ module and a structure theory for such modules was also developed in Propositions 1-5. Finally $(1-e) R(1-e)$ is a nilpotent finite ring and nilpotent finite rings were also studied in $\S 3$ of [11].

Note. I am deeply indebted to Prof. B. R. McDonald for his many detailed suggestions in regard to these and other results.

\section{REFERENCES}

1. G. Azumaya, On maximally central algebras, Nagoya Math. J., 2 (1951), 119-150.

2. W. E. Clark, A coefficient ring for finite noncommutative rings, Proc. Amer. Math. Soc., 33 (1972), 25-28.

3. C. W. Curtis and I. Reiner, Representation theory for finite groups and associative algebras, Interscience, 1962.

4. L. Fuchs, Ringe und ihre additive Gruppe, Publ. Math. Debrecen, 4 (1956), 488-508.

5. I. N. Herstein, Noncommutative rings, Carus Monograph, $\# 15,1968$.

6. E. Ingraham and F. DeMeyer, Separable Algebras Over Commutative Rings, Springer Verlag lecture notes in Mathematics, $\# 181,1971$.

7. N. Jacobson, Structure of Rings, Amer. Math. Soc. Colloq. Publ., XXXVII, 1964.

8. G. J. Janusz, Separable algebras over commutative rings, Trans. Amer. Math. Soc., 122 (1966), 461-479.

9. L. S. Levy, Torsion free and divisible modules over nonintegral domains, Canada. J. Math., 15 (1963), 132-152.

10. R. Raghavendran, Finite associative rings, Composition Math., 21 (1969), 195-229. 11. R. S. Wilson, On the structure of finite rings, Compositio Math., 26 (1973), 79-93.

Received November 2, 1972, and in revised form May 15, 1973. This paper is extracted from the author's thesis at the University of California at Santa Barbara where the author was an NSF Trainee working under the direction of Professor Adil Yaqub.

University of California, SANTA Barbara

AND

UNIVERSITY OF TEXAS 



\section{PACIFIC JOURNAL OF MATHEMATICS}

\section{EDITORS}

RICHARD ARENS (Managing Editor)

University of California

Los Angeles, California 90024

R. A. BeAumont

University of Washington

Seattle, Washington 98105
J. DugundJI*

Department of Mathematics

University of Southern California

Los Angeles, California 90007

D. Gilbarg and J. Milgram

Stanford University

Stanford, California 94305

\section{ASSOCIATE EDITORS}

E. F. BECKENBACH

B. H. NEUMANN

F. WOLF

K. YoSHIDA

\section{SUPPORTING INSTITUTIONS}

UNIVERSITY OF BRITISH COLUMBIA
CALIFORNIA INSTITUTE OF TECHNOLOGY
UNIVERSITY OF CALIFORNIA
MONTANA STATE UNIVERSITY
UNIVERSITY OF NEVADA
NEW MEXICO STATE UNIVERSITY
OREGON STATE UNIVERSITY
UNIVERSITY OF OREGON
OSAKA UNIVERSITY

UNIVERSITY OF BRITISH COLUMBIA CALIFORNIA INSTITUTE OF TECHNOLOGY UNIVERSITY OF CALIFORNIA MONTANA STATE UNIVERSITY NEW MEXICO STATE UNIVERSITY UNIVERSITY OF OREGON OSAKA UNIVERSITY
UNIVERSITY OF SOUTHERN CALIFORNIA STANFORD UNIVERSITY UNIVERSITY OF TOKYO UNIVERSITY OF UTAH WASHINGTON STATE UNIVERSITY UNIVERSITY OF WASHINGTON AMERICAN MATHEMATICAL SOCIETY NAVAL WEAPONS CENTER

* C. R. DePrima California Institute of Technology, Pasadena, CA 91109, will replace J. Dugundji until August 1974. 


\section{Pacific Journal of Mathematics}

\section{Vol. 51, No. $1 \quad$ November, 1974}

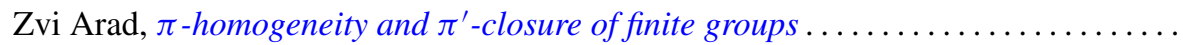

Ivan Baggs, A connected Hausdorff space which is not contained in a maximal

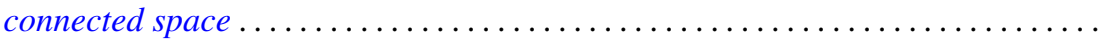

Eric Bedford, The Dirichlet problem for some overdetermined systems on the unit ball in $C^{n}$

R. H. Bing, Woodrow Wilson Bledsoe and R. Daniel Mauldin, Sets generated by

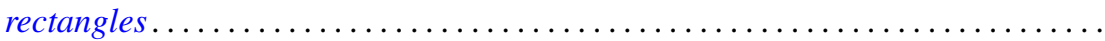

Carlo Cecchini and Alessandro Figà-Talamanca, Projections of uniqueness for

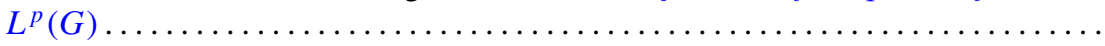

Gokulananda Das and Ram N. Mohapatra, The non absolute Nörlund summability of Fourier series .

Frank Rimi DeMeyer, On separable polynomials over a commutative ring ........ Richard Detmer, Sets which are tame in arcs in $E^{3} \ldots \ldots \ldots \ldots \ldots \ldots \ldots \ldots$

William Erb Dietrich, Ideals in convolution algebras on Abelian groups ..........

Bryce L. Elkins, A Galois theory for linear topological rings .................

William Alan Feldman, A characterization of the topology of compact convergence on $C(X)$.

Hillel Halkin Gershenson, A problem in compact Lie groups and framed cobordism

Samuel R. Gordon, Associators in simple algebras.

Marvin J. Greenberg, Strictly local solutions of Diophantine equations

Jon Craig Helton, Product integrals and inverses in normed rings . . . . . . . . . . . .

Domingo Antonio Herrero, Inner functions under uniform topology . . .

Jerry Alan Johnson, Lipschitz spaces .

Marvin Stanford Keener, Oscillatory solutions and multi-point boundary value

functions for certain nth-order linear ordinary differential equations.

John Cronan Kieffer, A simple proof of the Moy-Perez generalization of the

Shannon-McMillan theorem .......................

Joong Ho Kim, Power invariant rings

Gangaram S. Ladde and V. Lakshmikantham, On flow-invariant sets .

Roger T. Lewis, Oscillation and nonoscillation criteria for some self-adjoint even

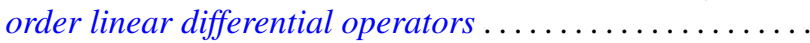

Jürg Thomas Marti, On the existence of support points of solid convex sets ..

John Rowlay Martin, Determining knot types from diagrams of knots . .

James Jerome Metzger, Local ideals in a topological algebra of entire functions

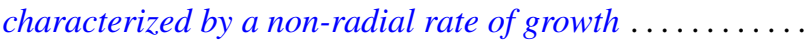

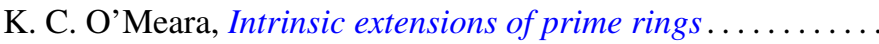

Stanley Poreda, A note on the continuity of best polynomial approximations ..

Robert John Sacker, Asymptotic approach to periodic orbits and local prolongations

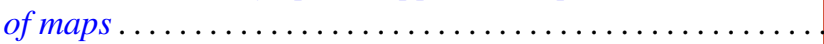

Eric Peter Smith, The Garabedian function of an arbitrary compact set . .

Arne Stray, Pointwise bounded approximation by functions satisfying a side condition

John St. Clair Werth, Jr., Maximal pure subgroups of torsion complete abelian

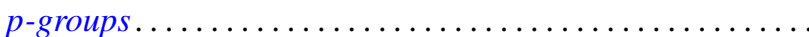

